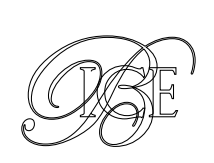

\title{
LA CRISIS DE LA COVID-19 Y LA ORGANIZACIÓN MUNDIAL DEL COMERCIO: ¿UNA OPORTUNIDAD?
}

La Organización Mundial del Comercio lleva años sumida en una profunda crisis en la que han intervenido factores como el papel de China o de Estados Unidos en la organización, el trato que reciben los países en vías de desarrollo, el aumento de acuerdos comerciales bilaterales y regionales o el bloqueo del Órgano de Apelación de su Sistema de Solución de Diferencias. A la crisis que atraviesa la OMC se suma, en estos momentos, la crisis sanitaria de la COVID-19: una pandemia sin precedentes que ha pillado desprevenidos tanto a Estados como a organizaciones internacionales y cuyas consecuencias van a perjudicar gravemente a las economías nacionales y al comercio mundial. No obstante, debemos plantearnos si este escenario puede suponer, al mismo tiempo, una oportunidad para abogar por el multilateralismo y reforzar la OMC, adaptar sus reglas a la situación actual y dar una respuesta global a un desafío global.

Palabras clave: coronavirus, pandemia, OMC, China, EE UU.

Clasificación JEL: F10, F50, F60.

\section{Introducción}

La organización multilateral por excelencia dedicada a regular los intercambios comerciales internacionales y compuesta en la actualidad por 164 Estados miembros, esto es, la Organización Mundial del Comercio (OMC), se encuentra sumida en una profunda crisis. Desde su creación en 1994, en el seno de la Ronda de Uruguay, la actividad de la OMC ha estado lastrada

* Investigadora predoctoral de la Junta de Castilla y León en la Universidad de Valladolid, área de Derecho Internacional Público y Relaciones Internacionales. Estudio cofinanciado por el Fondo Social Europeo.

Versión de abril de 2020.

DOI: https:/doi.org/10.32796/bice.2020.3125.7057 por la necesidad del consenso a la hora de adoptar decisiones y por la heterogeneidad de los Estados que la componen, todos ellos con diferentes puntos de vista e intereses. Sin embargo, en los últimos años, factores como el papel de China o Estados Unidos (EE UU) en la Organización, el trato que reciben los países en vías de desarrollo (PVD), el aumento de acuerdos comerciales bilaterales y regionales o el bloqueo del Órgano de Apelación (OA) han acentuado esta crisis hasta el punto de poner en entredicho la supervivencia de la OMC en el tiempo.

A la crisis que atraviesa la OMC se suma, en estos momentos, la crisis sanitaria de la COVID-19: una pandemia sin precedentes que ha pillado desprevenidos tanto a Estados $\triangle$ 
como a organizaciones internacionales y cuyas consecuencias van a perjudicar gravemente a las economías nacionales y al comercio mundial. No obstante, debemos plantearnos si este escenario puede suponer, al mismo tiempo, una oportunidad para abogar por el multilateralismo y reforzar el papel de la OMC, adaptar sus reglas a la situación actual y dar una respuesta global a un desafío global.

\section{La crisis actual de la Organización Mundial del Comercio}

En la actualidad, la OMC está compuesta por 164 Estados miembros, todos ellos con características muy heterogéneas, niveles de desarrollo muy diferentes e intereses contrapuestos, lo que hace más difícil y, en ocasiones, imposibilita adoptar decisiones por consenso y avanzar en la liberalización del comercio internacional. Esto fue, precisamente, lo que ocurrió en la Ronda de Doha del año 2001: la fragmentación de opiniones, intereses y puntos de vista entre los diferentes Estados miembros tuvieron como principal consecuencia la paralización y el fracaso de la agenda negociadora. Así, esta ronda de negociaciones se convirtió en la primera llamada de atención a los miembros de la OMC para que reflexionasen acerca de la pertinencia de una reforma estructural de la organización que permitiese avanzar en la consecución de sus objetivos y evitar, de esta manera, su fracaso. Sin embargo, esta crisis de la OMC se ha acentuado y hecho más evidente en los últimos años gracias a otra serie de factores.

\subsection{El papel de Estados Unidos}

De vital importancia en los últimos años ha sido la elección de Donald Trump, en noviembre del año 2016, como presidente de EE UU, muy escéptico en todo momento con el funcionamiento de la OMC y de su Sistema de Solución de Diferencias, a pesar de ser el miembro que más casos promueve ante el este sistema (Ávila, 2019: 28).

A este respecto, el representante comercial estadounidense, Robert Lighthizer, declaró en la última Conferencia Ministerial de la OMC, celebrada en Buenos Aires en diciembre de 2017, que, aunque es cierto que la OMC constituye una de las organizaciones internacionales más importantes, existen en la actualidad muchos retos que es necesario abordar, como la estructura y el funcionamiento del Sistema de Solución de Diferencias y del OA, la concesión del estatus de PVD, la falta de cumplimiento de las normas adoptadas o los problemas que originan las empresas de propiedad estatal ${ }^{1}$. Todos estos desafíos, según EE UU, tienen como principal protagonista a China, país con el que mantiene una guerra comercial abierta (Sanz, 2019: 80).

Además, dando cumplimiento a sus promesas electorales, Donald Trump, como supuesta «medida de seguridad nacional», ha incrementado los aranceles aduaneros en sectores de la economía estadounidense especialmente sensibles, como las industrias del acero y del aluminio, o la de los paneles solares, con la finalidad de protegerlas de la amenaza de importaciones más baratas procedentes de terceros Estados (Sampson, 2018: 465), lo que, claramente, constituye una política comercial proteccionista que Donald Trump defiende bajo el lema «America First» y que supone una amenaza al sistema multilateral de comercio internacional.

1 OMC: Declaración del Representante Comercial de EE UU, el Sr. Robert Lighthizer. 
Por último, la Administración Trump se ha caracterizado desde sus inicios por el abandono de negociaciones comerciales de carácter multilateral y regional y por la vuelta a la negociación de acuerdos comerciales bilaterales con terceros Estados, lo cual, en opinión de algunos autores, tendría como consecuencia principal el debilitamiento de la posición de EE UU en la escena global, tanto desde el punto de vista económico como desde el geopolítico, dado el carácter estratégico de los acuerdos megarregionales (Slominska y Waskinski, 2017: 98).

\subsection{China y la Organización Mundial del Comercio}

Como afirma Andrés González Martín, «China es la mayor ganadora de la globalización» (González, 2020: 1). Su envidiable crecimiento en las últimas décadas, en parte debido a la mejora en sus infraestructuras, a la creación de un gran tejido productivo, a un fuerte soporte tecnológico y al incremento de sus intercambios comerciales y relaciones diplomáticas con terceros Estados (González, 2020: 1), ha posicionado a China como una de las principales potencias económicas mundiales de primer orden, provocando el recelo del resto de los Estados más desarrollados. Sin embargo, las prácticas poco ortodoxas seguidas por China en el seno del sistema multilateral de comercio internacional es otra de las cuestiones que evidencian una crisis en el seno de la OMC y que supone una amenaza para el Sistema de Bretton Woods (Jannace y Tiffany, 2019: 1385).

Cuando China accedió a la condición de miembro de la OMC en diciembre del año 2001, estaba considerado como un Estado sin economía de mercado (dada la cantidad de empresas de propiedad estatal, los subsidios y las ayudas concedidas por parte del Gobierno y la intervención de este en diversos sectores), condición que habría de expirar pasados quince años, es decir, en diciembre de 2016. En la actualidad, China afirma ser una economía de mercado, pero la mayoría de Estados miembros de la OMC no parecen estar muy de acuerdo (Sampson, 2018: 468; Sanz, 2019: 81), dadas las evidencias de un incremento de la intervención estatal en la economía china y en las instituciones financieras (Jannace y Tiffany, 2019: 1390), siendo denominado este modelo como «capitalismo estatal chino» (Wu, 2015: 264). Este aspecto queda reflejado, asimismo, en el número de controversias presentadas ante el Órgano de Solución de Diferencias (OSD) de la OMC contra China².

Precisamente por ello, en la Conferencia Ministerial de Buenos Aires de 2017, las empresas de propiedad estatal o la definición de «economía de mercado» son temas recurrentes en las declaraciones de los representantes estatales cuando se abordan los desafíos a los que se enfrenta la OMC. Una de las cuestiones que más preocupa a países como EE UU es la protección de la propiedad intelectual e industrial, pues se estima que las pérdidas por la violación del Acuerdo sobre los Aspectos de los Derechos de Propiedad Intelectual relacionados con el Comercio por parte de China se pueden valorar en 600.000 millones de dólares americanos (González, 2020: 20). EE UU, concretamente, ha llegado a acusar a China de estar promoviendo o permitiendo intrusiones cibernéticas no autorizadas para hacerse con la tecnología y las patentes de las empresas occidentales (González, 2020: 19).

\footnotetext{
2 A fecha de abril de 2020 se han presentado 44 diferencias contra China ante la OMC. Vid. OMC: Diferencias en las que participa o ha participado China. https://www.wto.org/spanish/thewto_s/countries_s/ china_s.htm (última consulta: 5 de abril de 2020).
} 
Actualmente, China está tratando de alcanzar el liderazgo dentro de su mercado interno, pero también en el mercado global en un amplio espectro de tecnologías avanzadas relacionadas con las comunicaciones, la óptica o la defensa. En el logro de este objetivo, China ha emprendido importantes proyectos, incluyendo el controvertido «Made in China 2025», promulgado en el año 2015. La intención de esta ambiciosa iniciativa estratégica, bajo el liderazgo del primer ministro chino Li Keqiang, es transformar a China, actual proveedor de mano de obra barata $y$ poco cualificada, en un Estado capaz de diseñar y producir bienes y servicios innovadores, competitivos y de alto valor añadido en tecnologías de vanguardia, capaces de dominar la economía global del futuro (Jannace y Tiffany, 2019: 1394; González, 2020: 14). El objetivo principal del Made in China 2025 es que la producción local de componentes básicos en China aumente del $40 \%$ en el año 2020 al $70 \%$ en el año 2025 (González, 2020: 18). De manera complementaria, con la iniciativa de la Nueva Ruta de la Seda, China pretende evitar la sobrecarga de su industria básica y garantizar el acceso a las materias primas necesarias en sus procesos productivos (González, 2020: 14).

La rápida transformación de la economía china y su sorprendente capacidad exportadora la han convertido en una de las fábricas mundiales junto a otros países como India. No obstante, su forma de proceder en el comercio mundial supone, en palabras de Andrés González Martín, una «grave patología» que terminará perjudicando el proceso globalizador. En este contexto, «la única alternativa es disciplinar económicamente $-\mathrm{y}$ comercialmente- al gigante asiático» (González, 2020: 1).

\subsection{El trato especial y diferenciado a los países en vías de desarrollo}

Otro de los desafíos a los que se enfrenta la OMC en el trato ofrecido en su seno a los PVD, a quienes frecuentemente se les presenta como los grandes perjudicados de la globalización, dada la desigual participación de estos en el comercio mundial y la vulnerabilidad de sus economías en las crisis financieras internacionales (López-Jurado, 2001: 1-2).

En el año 1986, al inicio de la Ronda de Uruguay que da vida a la OMC, la Declaración de Punta del Este ${ }^{3}$ establece que, durante las negociaciones de la Ronda, se evitará el desmantelamiento del principio de trato diferenciado y más favorable a los PVD, recogido en la Parte IV del Acuerdo General sobre Aranceles Aduaneros y Comercio (GATT por sus siglas en inglés). Finalmente, el Preámbulo del Acuerdo de Marrakech reconoce que «es necesario realizar esfuerzos positivos para que los países en desarrollo, y especialmente los menos adelantados, obtengan una parte del incremento del comercio internacional que corresponda a las necesidades de su desarrollo económico» ${ }^{4}$. Asimismo, el acuerdo recoge todas las prerrogativas concedidas a esta serie de países en el GATT de 1947, reafirmando el trato especial y diferenciados a los PVD en el seno de la OMC. En la actualidad, más de dos tercios de los 164 miembros de la OMC se consideran a sí mismos como países en desarrollo (Sanz, 2019: 78) y existen 148 disposiciones relativas al trato especial y diferenciado a los PVD en los acuerdos de la OMC, destinadas, todas ellas, a dotar de flexibilidad legal a los intercambios $\square$

\footnotetext{
3 Declaración de Punta del Este, de 20 de septiembre de 1986. http:// www.sice.oas.org/trade/Punta_s.asp (última consulta: 6 de abril de 2020).

4 Acuerdo de Marrakech por el que se establece la Organización Mundial del Comercio, Marrakech, de 15 de abril de 1994 (LT/UR/A/2, de 15 de abril de 1994, p. 11).
} 
comerciales de los Estados menos desarrollados (Sampson, 2018: 461).

Una vez reconocida la desigual situación y el papel de los PVD en el sistema de comercio multilateral en los textos fundacionales de la OMC, ahora el desafío es defender su posición en las negociaciones que se realizan en el seno de la organización, siendo en este punto en el que se encuentran las mayores dificultades al contar los países más pobres con una débil representación, pues, como explica el profesor Lobejón Herrero, «a muchos de ellos no les sirve de nada disponer de un voto en la mayor parte de las instancias de la OMC, ya que, o bien no tienen representación en Ginebra - porque no pueden costearla- o es muy reducida, lo que les impide asistir a todas las reuniones que se celebran simultáneamente». Además, «el problema se ve agravado por el hecho de que sus funcionarios carecen de formación adecuada para participar en los complejos mecanismos que gestiona la OMC» (Lobejón, 2010: 82).

Unido a la débil representación de los PVD en los diferentes órganos de la OMC, otro desafío añadido es el hecho de que la categoría de PVD no es homogénea, no existe una diferenciación entre niveles de desarrollo (Sanz, 2019: 78), pudiendo diferenciarse dentro de esta categoría distintos subgrupos. En opinión de la profesora López-Jurado (2001:2), este hecho «apunta hacia la necesidad de elaborar un estatuto jurídico comercial particular para cada uno de ellos y, desde una perspectiva mucho más amplia, a la necesidad de reformular el sistema internacional de ayuda al desarrollo", pues una de las principales razones de la falta de efectividad de muchas de las previsiones «legalmente flexibles» en favor de los PVD es, precisamente, que estas son genéricas para todos los países en desarrollo y se olvidan de las especificidades de cada uno de ellos (Sampson, 2018: 461).

En la Ronda de Doha del año 2001, todas las quejas de los PVD fueron tenidas en cuenta y se acordó revisar las disposiciones relativas al trato especial y diferenciado «con miras a reforzarlas y hacerlas más precisas, eficaces y operativas» 5 . No obstante, no ha habido progresos en las negociaciones en relación a este objetivo (Sampson, 2018: 462).

\subsection{El incremento de los acuerdos comerciales bilaterales y regionales}

En la actualidad, estamos asistiendo a un cambio de paradigma. Si durante el tiempo de vigencia del GATT de 1947 la regla general era la cláusula de nación más favorecida, según la cual las ventajas o beneficios arancelarios concedidos a una de las partes contratantes del GATT habrían de ser extendidas al resto de partes contratantes, y la excepción eran las uniones aduaneras y las zonas de libre comercio, hoy esto se ha invertido, en parte como consecuencia de la dificultad de avanzar en las negociaciones en el marco de la OMC, y la mayor parte del comercio mundial se rige por acuerdos de libre comercio bilaterales o regionales, a través de los que los Estados parte del acuerdo eliminan mutuamente las barreras arancelarias y no arancelarias que entre ellos pudiesen existir (Fernández, 2016: 125). Hemos pasado, como bien dice Ana Manero Salvador, de un marco comercial multilateral, más o menos previsible, a una atomización de las relaciones comerciales a través de acuerdos de libre comercio, muchos de ellos $\square$

5 Párrafo 44 de la Declaración Ministerial de Doha, de 20 de noviembre de 2001 (WT/MIN(01)/DEC/1, de 20 de noviembre de 2001, p. 10). 
bilaterales (Manero, 2018: 29), originándose, de esta manera, una fragmentación del comercio mundial.

Respecto de este fenómeno, existen opiniones encontradas. Por un lado, algunos autores afirman que este tipo de acuerdos son como «termitas» que restan legitimidad y suponen una amenaza a la continuidad del sistema multilateral de comercio internacional y los principios rectores elementales de este sistema (Bhagwati, 2008), mientras que otros autores defienden que, aunque es cierto que la naturaleza de estos acuerdos es discriminatoria, los acuerdos de libre comercio constituyen un instrumento de gran importancia para los Estados que desean avanzar en el proceso de liberalización del comercio mundial (Sampson, 2018: 457).

\subsection{La crisis del Sistema de Solución de Controversias y del Órgano de Apelación}

El quinto de los problemas a los que se enfrenta la OMC en la actualidad, y, quizá, el más importante en estos momentos de cara a su supervivencia en un futuro, se encuentra relacionado con el OA previsto en el Sistema de Solución de Diferencias.

EI OA es un órgano de carácter permanente y cuasijudicial cuya misión es revisar en apelación los informes de los Grupos de Expertos cuando las partes en una controversia comercial así lo desean, dotando al Sistema de independencia, seguridad y de una interpretación consistente del Derecho de la OMC (Arredondo y Godio, 2019: 167; Sanz, 2019: 76). Este órgano está compuesto por siete individuos de reconocido prestigio y competencia acreditada en derecho y comercio internacional (no tienen por qué ser jueces per se), que desarrollan su título de manera independiente a las directrices de sus Gobiernos, y que son elegidos por periodos de cuatro años con la posibilidad de ser reelegidos por periodos de igual duración. A su vez, el OA se divide en tres subgrupos integrados por tres miembros elegidos aleatoriamente para conocer los casos que ante él se presenten (García-Matamoros y Arévalo-Ramírez, 2018: 681682; Arredondo y Godio, 2019: 164 y 168).

El problema con el OA es que, en junio del año 2017, por cuestiones formales de rotación de los individuos que componen el OSD, se produjeron tres vacantes que no han podido ser cubiertas por la falta de consenso en el seno del mismo y, más concretamente, por el bloqueo de EE UU (Arredondo y Godio, 2019: 169). En septiembre del año 2018, el OA alcanzó el mínimo de miembros para poder considerar la apelación, esto es, tres individuos. Finalmente, el 11 de diciembre del año 2019 expiró el mandato de dos miembros más: el indio Ujal Singh Bhatia y el estadounidense Thomas R. Graham, dejando el OA compuesto únicamente por la china Hong Zhao ${ }^{6}$. No obstante, en una comunicación del 10 de diciembre de $2020^{7}$, el Sr. Graham notificó al presidente del OSD que los tres miembros del OA proseguirían los trabajos necesarios en relación con las apelaciones respecto de las cuales se hubiera completado una audiencia.

Este bloqueo en la renovación de los miembros del OA y el cese de sus funciones suponen un riesgo sistémico importante, pues, ante la falta de un informe y de unas recomendaciones imparciales, puede ocurrir que la parte $D$

6 OMC: Miembros del Órgano de Apelación. https://www.wto.org/ spanish/tratop_s/dispu_s/ab_members_descrp_s.htm (última consulta: 9 de abril de 2020)

7 OMC: Comunicación del Presidente del Órgano de Solución de Diferencias, de 10 de diciembre de 2020 (WT/DSB/79, de 12 de diciembre de 2019). 
afectada por la controversia decida recurrir a represalias determinadas unilateralmente $\sin$ ningún tipo de control (Sampson, 2018: 670). De esta manera, las próximas controversias relativas a cuestiones comerciales internacionales se resolverán utilizando la ley del más fuerte.

En este contexto, conviene analizar las razones por las cuales EE UU mantiene bloqueada la renovación del OA. En primer lugar, destaca su desacuerdo con la denominada «Regla 15» adoptada por el propio OA y contenida en los procedimientos de trabajo para el examen en apelación ${ }^{8}$, según la cual «una persona que deje de ser miembro del OA podrá, con autorización del OA y previa notificación al OSD, terminar la sustanciación de cualquier apelación a la que hubiera sido asignada cuando era miembro y, a tal efecto, únicamente, se considerará que sigue siendo miembro del $O A$ » con el objeto de dar continuidad y coherencia a las diferencias sometidas a una revisión en apelación. EE UU considera que esta regla es contraria al Acuerdo sobre sobre el Entendimiento relativo a las Normas y Procedimientos por los que se rige la Solución de Diferencias, que prevé tiempos máximos para los mandatos de los jueces del OA, y que, en todo caso, debería ser el OSD quien aprobase las extensiones de dichos mandatos en el tiempo (Arredondo y Godio, 2019: 171). Así mismo, EE UU argumenta que la Regla 15 y el documento relativo a procedimientos de trabajo para el examen en apelación del año 2010 no forman parte del Acuerdo relativo al Entendimiento para la Solución de Diferencias y, por tanto, no constituye una norma aprobada por los Estados miembros de la OMC (Arredondo y Godio, 2019: 172). En

8 OMC: Procedimientos de trabajo para el examen en apelación, de 16 de agosto de 2010 (WT/AB/WP/6, de 16 de agosto de 2010). segundo lugar, otra de las críticas que EE UU formula contra el OA se encuentra relacionada con la lentitud de emisión de sus informes. A tenor de lo dispuesto en el art. 17 del Acuerdo sobre Entendimiento para la Solución de Diferencias, el OA debe emitir el informe en apelación en un plazo de 60 días, prorrogable hasta 90 días. No obstante, en los últimos años estos plazos se han ampliado como consecuencia de un aumento de las demandas y de la mayor complejidad de las diferencias, no viéndose aumentado el número de individuos que componen el OA (Sanz, 2019: 77). Por último, otra de las críticas de EE UU al OA es que a la hora de elaborar sus informes incluye interpretaciones innecesarias para la solución de la diferencia, que, incluso, atañen a las legislaciones internas de los Estados. Según EE UU, estas interpretaciones configuran paulatinamente un cuerpo legal que no ha sido negociado ni aprobado por los Estados miembros de la OMC (Sanz, 2019: 77).

Desde el año 2018 se vienen estudiando y presentando propuestas de reforma de la OMC, en las que la revisión del Sistema de Solución de Diferencias y del OA es central. En mayo de 2018, un grupo de Estados, entre los que se incluía la UE, propusieron, «habida cuenta de la urgencia y la importancia de cubrir los puestos vacantes en el OA, de conformidad con el Acuerdo relativo al Entendimiento para la Solución de Diferencias, y con el fin de que pueda desempeñar adecuadamente sus funciones", activar el proceso de nombramiento de nuevos jueces para el $\mathrm{OA}^{9}$, a lo que EE UU se negó en un comunicado de 22 de junio ${ }^{10}$. Más adelante, en octubre de 2018, Canadá $\triangleright$

\footnotetext{
9 OMC: Nombramiento de Miembros del Órgano de Apelación, de 17 de mayo de 2018 (WT/DSB/W/609/Rev.4, de 18 de mayo de 2018).

10 U.S. Statements at the June 22, 2018, DSB Meeting. https://geneva. usmission.gov/wp-content/uploads/sites/290/Jun22.DSB_.Stmt_.asdelivered.fin_.public.rev_.pdf
} 
convocó una reunión de alto nivel para intentar alcanzar soluciones a la que ni China ni EE UU fueron invitados para no enturbiar los debates, en la que, finalmente, se puso de manifiesto la importancia de desbloquear el nombramiento de nuevos jueces del OA para mantener la OMC operativa ${ }^{11}$. De gran importancia fue la Comunicación de la Unión Europea, China, Canadá, la India, Noruega, Nueva Zelanda, Suiza, Australia, República de Corea, Islandia, Singapur y México, que habría de ser presentada al Consejo General de la OMC el 12 de diciembre de $2018^{12}$. En esta comunicación, «preocupados porque la falta de consenso en el OSD para cubrir las vacantes del OA socave la viabilidad del sistema de solución de diferencias de la OMC», este grupo de Estados propone modificaciones relativas a Normas de Transición para los miembros salientes del Órgano de Apelación, plazos de emisión de informes, el sentido del derecho interno como una cuestión de hecho y las constataciones innecesarias para la solución de la diferencia, entre otros. A día de hoy, ninguna de estas propuestas ha sido formalmente aceptada.

\section{La crisis de la COVID-19: ¿una oportunidad?}

Al tiempo que se escriben estas líneas, la autora se encuentra confinada en su domicilio como consecuencia del estado de alarma decretado por el Gobierno de España ${ }^{13}$ por la

\footnotetext{
11 Joint Communiqué of the Ottawa Ministerial on WTO Reform, de 24 y 25 de octubre de 2018. https://www.canada.ca/en/global-affairs/ news/2018/10/joint-communique-of-the-ottawa-ministerial-on-wto-reform. html (última consulta: 11 de abril de 2020).

12 OMC: Comunicación de la Unión Europea, China, el Canadá, la India, Noruega, Nueva Zelandia, Suiza, Australia, la República de Corea, Islandia, Singapur y México al Consejo General, de 23 de noviembre de 2018 (WT/GC/W/752, de 26 de noviembre de 2018).

13 Real Decreto 463/2020, de 14 de marzo, por el que se declara el estado de alarma para la gestión de la situación de crisis sanitaria
}

crisis sanitaria ocasionada por el coronavirus 2019. El pasado 11 de marzo del año 2020 el director general de la Organización Mundial de la Salud (OMS), el etíope Tedros Adhanom Ghebreyesus, declaró que la enfermedad denominada "COVID-19», con origen en la ciudad de Wuhan, China, había alcanzado el nivel de pandemia mundial ${ }^{14}$, dados los alarmantes niveles de propagación y gravedad, haciendo, al mismo tiempo, un llamamiento a los países para que adoptasen medidas «urgentes y agresivas». En estos momentos, la mayoría de los Estados han implementado medidas de confinamiento domiciliario de cara a contener la propagación de los contagios y muchos sectores de la economía han visto suspendida su actividad. Según el secretario general de Naciones Unidas, António Guterres, «estamos enfrentando una crisis de salud global como ninguna anterior en los 75 años de historia de la Organización de Naciones Unidas: una que está propagando el sufrimiento humano, infectando la economía global y el cambio de las vidas de las personas» ${ }^{15}$.

La crisis de la COVID-19 también ha tenido repercusión en el seno de la OMC, que se ha visto obligada a suspender el proceso de acreditación de los medios de comunicación y de las ONG para la Duodécima Conferencia Ministerial (CM12) prevista del 8 al 11 de junio en Kazajstán ${ }^{16}$, por si, finalmente, esta ha de ser cancelada. El presidente del Consejo General $\triangleright$

ocasionada por el COVID-19 (BOE 67, de 14 de marzo de 2020, pp. 25390-25400).

14 OMS: Alocución de apertura del Director General de la OMS en la rueda de prensa sobre la COVID-19 celebrada el 11 de marzo de 2020. https://www. who.int/es/dg/speeches/detail/who-director-general-sopening-remarks-at-the-media-briefing-on-covid-19---11-march-2020 (última consulta: 11 de abril de 2020).

15 ONU: Secretary-General Remarks on COVID-19: A Call for Solidarity, de 19 de marzo de 2020. https://www.un.org/sites/un2.un.org/ files/sg_remarks_on_covid-19_english_19_march_2020.pdf

16 OMC:Suspensión de la acreditación de los medios de comunicación y de las ONG para la Duodécima Conferencia Ministerial. $h$ ttps://www.wto. org/spanish/news_s/news20_s/mc12_24mar20_s.htm (última consulta: 11 de abril de 2020). 
de la Organización, David Walker, tras una serie de consultas con los Estados miembros, se ha comprometido a organizar, cuando las condiciones lo permitan, una reunión extraordinaria del Consejo General para que los miembros puedan reflexionar sobre la mejor manera de proceder al respecto ${ }^{17}$. El papel de la OMC en esta crisis, no actualmente, sino cuando la propagación de la pandemia consiga controlarse, será de vital importancia, dado que va a perjudicar gravemente al pilar fundamental que mantiene la estructura de la economía mundial: el comercio internacional (Frías, 2020: 5). Esta crisis sanitaria mundial está poniendo en duda la razón de ser de la deslocalización empresarial y la fe en un comercio internacional liberalizado y sin barreras. La gran dependencia del mercado exterior, en el caso de algunos Estados, y el hecho de que, en la actualidad, la producción de algunos elementos sanitarios básicos, como mascarillas, trajes de protección o respiradores artificiales, se encuentre radicada en países, principalmente, del continente asiático y esté sufriendo trabas para llegar a los países de destino, lo que se denomina comúnmente como rotura de las cadenas de abastecimiento (Laborie, 2020: 14), está haciendo aflorar la idea de la pertinencia de la reindustrialización de determinados sectores estratégicos de las economías nacionales, aunque económicamente resulte menos rentable (Frías, 2020: 6). Además, si finalmente se opta por la reindustrialización de estos sectores estratégicos, es más que probable el repunte de las guerras comerciales internacionales en las que los Estados con menos capacidad financiera serán los principales perjudicados (Laborie, 2020: 14).

17 OMC: Duodécima Conferencia Ministerial de la OMC. $h t t p s: / / w w w$. wto.org/spanish/thewto_s/minist_s/mc12_s/mc12_s.htm (última consulta: 11 de abril de 2020).
Así, aunque el escenario futuro que esta pandemia mundial nos va a dejar es totalmente incierto, si algo es seguro, es que las economías de todos los Estados, independientemente del nivel de desarrollo, se van a ver significativamente mermadas, como siempre ha ocurrido tras una catástrofe de índole mundial $^{18}$ y que asistiremos a un profundo cambio del orden mundial actual. Para hacer frente al desafío, los Estados tienen dos opciones: o bien abogar por el aislacionismo, el unilateralismo y por políticas económicas y sociales de corte proteccionista ${ }^{19}$ y volver, de esta manera, al mundo westafaliano previo a 1945 de soberanismo nacional y de Estado-nación (Márquez, 2020: 9), o, por el contrario, apostar por el refuerzo de las instituciones internacionales de carácter multilateral, lo cual implica una clara disposición para cooperar entre el mayor número de Estados posible aceptando reglas comunes, y su, en palabras del profesor Remiro Brotóns (1999:14), "superior bondad", e intentar construir un nuevo orden internacional, regulado por organizaciones internacionales $\triangleright$

18 En su Informe sobre las Perspectivas de la Economía Mundial del 14 de abril del año 2020, el FMI ha pronosticado, para lo que queda de año, una caída del producto interior bruto mundial del $3 \%$, siendo España una de las economías más afectadas con una caída en su PIB de un $8 \%$, solo por detrás de la economía italiana, que decrecerá un $9,1 \%$. Sorprendentemente, para China, epicentro de la crisis sanitaria, el FMI prevé un crecimiento del $1,2 \%$. Más información en Últimas proyecciones de crecimiento de Perspectivas de la Economía Mundial del Fondo Monetario Internacional, de 14 de abril de 2020. https://www.imf.org/es/Publications/WEO/Issues/2020/04/14/weoapril-2020\#Introducci\%C3\%B3n (última consulta: 14 de abril de 2020).

19 Antes de la llegada de la crisis de la COVID-19, el panorama mundial se encontraba gobernado por el ascenso de los populismos, un creciente rechazo a la globalización y la vuelta a las medidas económicas de carácter proteccionista, protagonizadas por EE UU. La reacción inicial a la pandemia mundial, en palabras de Márquez de la Rubia, «acentuó todas estas tendencias", provocando el cierre de fronteras, una disminución preocupante del comercio mundial y el cuestionamiento de la eficacia tanto de los Gobiernos nacionales como de las organizaciones internacionales (Márquez, 2020: 3).

En el caso particular de la UE, la crisis del coronavirus 2019 ha evidenciado - aún más si cabe - la fragilidad de esta organización internacional de carácter regional, ya mermada por el Brexit, así como los diferentes intereses de los Estados que la componen. Como afirma Frías Sánchez (2020:12-13), «si la UE fracasa también en la gestión de las consecuencias económicas de la crisis del coronavirus, el proceso de integración europea puede sufrir un golpe mucho mayor que el que supondrá el brexit». 
de carácter multilateral, liderado por potencias y dinamizado por la sociedad civil y los sujetos privados (Peredo, 2020: 13).

Si finalmente se apuesta por el multilateralismo, la OMC puede hacer de la necesidad virtud y salir especialmente reforzada de esta situación si sus Estados miembros deciden consensuar nuevas normas adaptadas a la realidad actual y aportar soluciones determinantes a los problemas y a la falta de eficacia que viene arrastrando desde hace ya décadas, y es que «el multilateralismo es eficaz porque es legítimo per se» (Barbé, 2010: 35). El deterioro de los intercambios comerciales internacionales tras la crisis de la COVID-19 puede ser corregido más fácilmente por una organización internacional de carácter multilateral con una respuesta única que por la actuación individual de numerosos Estados con intereses contrapuestos en la búsqueda del liderazgo mundial.

\section{Conclusiones}

En el pasado, los grandes cambios en el orden internacional general tuvieron como origen los grandes conflictos bélicos y las crisis económicas. La fe en el multilateralismo, en la cooperación internacional y en las organizaciones internacionales de vocación universal fue determinante hace casi un siglo para superar el desastre causado por una de estas guerras, la Segunda Guerra Mundial, y fue, asimismo, fundamental para la recuperación tras la crisis financiera del año 2008 y, en esta ocasión, puede constituir un remedio a los estragos económicos y comerciales que la COVID-19 pueda causar en un futuro ante la imposibilidad a corto plazo de que EE UU y China resuelvan sus diferencias y decidan entablar lazos de cooperación estables.
La OMC debe hacer de la necesidad virtud $y$, en un alarde de resiliencia, tomar esta situación como una oportunidad para, finalmente, actuar con determinación e independencia, adaptar sus normas y principios comerciales básicos a la realidad existente y tratar de solucionar los problemas que la han llevado a la crisis en la que se halla sumida desde hace años. La pandemia mundial de la COVID-19 y sus consecuencias en la economía y en el comercio internacional requieren de una respuesta igualmente mundial, que no puede venir sino de la mano de una organización comercial de carácter multilateral como lo es la OMC.

\section{Bibliografía}

Arredondo, R., y Godio, L. M. A. (2019). La Crisis del Órgano de Apelación de la Organización Mundial de Comercio. Revista Da Secretaria Do Tribunal Permanente de Revisão, 7(13), 163-179. https://doi.org/10.16890/rstpr.a7.n13.p163

Ávila, A. M. (2019). La evolución del Sistema de Solución de Diferencias de la OMC. Boletín Económico de ICE (3116), 19-29. https:/doi.org/10.32796/ bice.2019.3116.6915

Barbé Izuel, E. (2010). Multilateralism: Adapting to World with Emerging Powers. Revista Española de Derecho Internacional, 62(2), 21-50.

Bhagwati, J. (2008). Termites in the Trading System: How Preferential Agreements Undermine Free Trade. Oxford: Oxford University Press.

Fernández Carbajal, A. (2016). Los Acuerdos Comerciales Exteriores de la Unión Europea: España ante el Acuerdo de Libre Comercio UE-Corea del Sur. Cuadernos Jean Monnet sobre integración europea fiscal y económica (2), 123-138.

Frías Sánchez, C. J. (2020). Paisaje después de la batalla: el mundo tras el COVID-19. Documento de Opinión IEEE 27/2020. 
García-Matamoros, L. V., y Arévalo-Ramírez, W. (2018). La Actividad del Sistema de Solución de Diferencias de la Organización Mundial del Comercio: su Eficacia, Limitaciones y Uso Diferenciado por Países en Vías de Desarrollo y Países Desarrollados. En H. Olasolo et al. (coords.), Alcance y Limitaciones de la Justicia Internacional. Tirant Lo Blanch.

González Martín. (2020). La globalización está en cuarentena. Documento de Análisis IEEE 11/2020, 1-27.

Jannace, W., y Tiffany, P. (2019). A New World Order: The Rule of Law, or the Law of Rulers. Fordham International Law Journal, 42(5), 1379-1418.

Laborie, M: (2020). Desglobalización y pandemia global. Documento de Opinión IEEE 28/2020.

Lobejón Herrero, L. F. (2010). Más allá de la Ronda de Doha. El futuro de la OMC. Papeles de Relaciones Ecosociales y Cambio Global (112), 81-88.

López-Jurado, C. (2001). El Tratamiento de los países en vías de desarrollo en la OMC y las iniciativas unilaterales de la Comunidad Europea. Revista Electrónica de Estudios Internacionales (3), 1-38.

Manero Salvador, A. (2018). Los Tratados de Libre Comercio de Estados Unidos y de la Unión Europea. Bosch Editor.
Márquez De La Rubia, F. (2020). Ante la pandemia: un renovado liderazgo global. Documento de Opinión IEEE 29/2020.

Peredo Pombo, J. M. (2020). El orden internacional y el necesario refuerzo de la Alianza Trasatlántica tras la pandemia del COVID-19. Documento de Opinión IEEE 26/2020.

Remiro Brotóns, A. (1999). Universalismo, multilateralismo, regionalismo y unilateralismo en el nuevo orden internacional. Revista Española de Derecho Internacional, 51(1), 11-57.

Sampson, G. P. (2018). Challenges Facing the World Trade Organization: An Overview. Australian Economic Review, 51(4), 453-473. https:/doi. org/10.1111/1467-8462.12301

Sanz Serrano, A. (2019). Estados Unidos y el Sistema Multilateral de Comercio. Boletín Económico de ICE (3110), 75-84. https://doi.org/10.32796/ bice.2019.3110.6789

Slominska, B., y Wasinski, M. (2017). The Prospects for U.S. Trade Policy under the Trump Administration. The Polish Quarterly of International Affairs, 26(1), 83-98.

Wu, M. (2016). The «China, Inc.» Challenge to Global Trade Governance. Harvard International Law Journal, 57(2), 261-324. 
